hep-th/9410178

MPI-PhT/94-65

ZU-TH 31/94

October 1994

\title{
FERMIONS IN THE BACKGROUND OF DILATONIC SPHALERONS
}

\author{
GEORGE LAVRELASHVILI \\ Institut für Theoretische Physik, Universität Zürich \\ Winterthurerstrasse 190, CH-8057 Zürich, Switzerland \\ and \\ Max-Planck-Institut für Physik, Werner-Heisenberg-Institut \\ Föhringer Ring 6, D-80805 Munich, Germany
}

\begin{abstract}
We discuss the properties and interpretation of a discrete sequence of a static spherically symmetric solutions of the Yang-Mills-dilaton theory. This sequence is parametrized by the number $n$ of zeros of a component of the gauge field potential. It is demonstrated that solutions with odd $n$ posses all the properties of the sphaleron. It is shown that there are normalizable fermion zero modes in the background of these solutions. The question of instability is critically analysed.
\end{abstract}

*Electronic address: lavrela@physik.unizh.ch

$\dagger^{\dagger}$ On leave of absence from Tbilisi Mathematical Institute, 380093 Tbilisi, Georgia

${ }^{\ddagger}$ Address after October 1, 1994. 


\section{Introduction}

A discrete sequence of static finite energy solutions of a Yang-Mills-dilaton (YMD) theory was found recently [1] (see also [2]). These solutions can be labeled by the number of zeros of the gauge function and have the same nature as the BartnikMcKinnon (BMK) solutions [3] of the coupled Einstein-Yang-Mills (EYM) equations. On the other hand both the BMK solutions and the solutions of the YMD theory are related to usual electroweak sphaleron [4], [5], [6], [7].

The sphaleron solution was found in ref. [4] and later was interpreted [5], [6] as a solution, which lies on the top of the energy barrier separating topologically different vacua [B]. Creation and subsequent decay of the sphaleron leads to the fermion number non-conservation [9] related to the existence of the Adler-Bell-Jackiw (ABJ) anomaly [10]. This fermion number non-conservation has natural interpretation in terms of the phenomenon of fermion level crossing (see e.g., [11] and ref. therein) in the background of a topologically nontrivial gauge field. The observation is that a fermion zero mode occurs, when the gauge field passes the sphaleron configuration.

The solutions of YMD theory with an odd number of zeros of the gauge function carry all the main properties of the sphaleron, namely:

(i) they have finite energy

(ii) they are saddle points of the action functional

(iii) they have fractional topological charge

(iv) there are fermion zero modes in background of these solutions.

Therefore it is natural to interpret them as a dilatonic sphalerons.

In the present paper we will critically review the stability analysis and demonstrate properties (iii) and (iv) of the dilatonic sphalerons.

The rest of the paper is organized as follows. In the next section we will review dilatonic sphaleron solutions. In section 3 we will show that similarly as for the electroweak sphaleron one can assign a (half-integer) topological charge to the dilatonic sphalerons. In section 4 we will demonstrate existence of the fermion zero modes in the background of the dilatonic sphalerons. In section 5 we will present the stability analysis. Section 6 contains concluding remarks.

\section{Dilatonic Sphalerons}

We are interested in YMD theory defined by the action

$$
S=\frac{1}{4 \pi} \int d^{4} x\left(\frac{1}{2}\left(\partial_{\mu} \varphi\right)\left(\partial^{\mu} \varphi\right)-\frac{\mathrm{e}^{2 \kappa \varphi}}{4 g^{2}} F_{\mu \nu}^{a} F^{a \mu \nu}\right)
$$

where $F_{\mu \nu}^{a}$ is a $S U(2)$ gauge field strength $F_{\mu \nu}^{a}=\partial_{\mu} W_{\nu}^{a}-\partial_{\nu} W_{\mu}^{a}+\epsilon^{a b c} W^{b} W_{\nu}^{c}$ and $a=1,2,3$ is $S U(2)$ group index, $\mu, \nu=0,1,2,3$ are space-time indexes. 1 Using scaling properties of the action Eq.(1) one can remove the dependence on the dilatonic

\footnotetext{
${ }^{1}$ Our conventions are as follows: $A_{\mu} B^{\mu}=A_{0} B^{0}+A_{i} B^{i}=A_{0} B_{0}-A_{i} B_{i}$ where $i, j=1,2,3$ are space indexes.
} 
coupling constant $\kappa$ and the gauge coupling constant $g$ from $S$, therefore we may put $\kappa=g=1$ in what follows without restriction.

We are interested in spherically symmetric solutions. For our analysis we will essentially adopt the notations of refs. [12], [13]. The most general spherically symmetric ansatz for the $S U(2)$ Yang-Mills field $W_{\mu}^{a}$ can be written (in the Abelian gauge) as 114

$$
\begin{array}{ll}
W_{t}^{a}=\left(0,0, A_{0}\right), & W_{\theta}^{a}=\left(\phi_{1}, \phi_{2}, 0\right) \\
W_{r}^{a}=\left(0,0, A_{1}\right), & W_{\varphi}^{a}=\left(-\phi_{2} \sin \theta, \phi_{1} \sin \theta, \cos \theta\right) .
\end{array}
$$

This ansatz Eq.(2) is form invariant under gauge transformations around the third isoaxis, with $A_{\alpha}$ transforming as a $U(1)$ gauge field on the reduced space-time $(t, r)$, whereas $\phi=\phi_{1}+i \phi_{2}$ is a scalar of charge one with the covariant derivative $D_{\alpha} \phi=$ $\partial_{\alpha} \phi-i A_{\alpha} \phi$. With respect to this $U(1)$ one may define the 'charge conjugation' $\phi \rightarrow \bar{\phi}$, $A_{\alpha} \rightarrow-A_{\alpha}$. The corresponding YMD action is

$$
S=\int d r d t\left[\frac{r^{2}}{2}\left(\partial_{\alpha} \varphi\right)\left(\partial^{\alpha} \varphi\right)-\mathrm{e}^{2 \varphi}\left(\frac{r^{2}}{4} F^{\alpha \beta} F_{\alpha \beta}-\overline{D^{\alpha} \phi} D_{\alpha} \phi+\frac{1}{2 r^{2}}\left(|\phi|^{2}-1\right)^{2}\right)\right],
$$

where $F_{\alpha \beta}$ denotes the field strength of $A_{\alpha},(\alpha, \beta=t, r)$.

Choosing the gauge $A_{0}=0$ the YMD action Eq.(3) can be written more explicitly

$$
S=\int d r d t\left[\frac{r^{2}}{2} \dot{\varphi}^{2}-\frac{r^{2}}{2} \varphi^{\prime 2}-\mathrm{e}^{2 \varphi}\left(-\frac{r^{2}}{2} \dot{A}_{1}^{2}-|\dot{\phi}|^{2}+\left|\phi^{\prime}-i A_{1} \phi\right|^{2}+\frac{1}{2 r^{2}}\left(|\phi|^{2}-1\right)^{2}\right)\right]
$$

where a prime denotes $\frac{d}{d r}$ and a dot $\frac{d}{d t}$.

It was found [1], [2] that the YMD theory in the even sector with respect to $U(1)$ charge conjugation,

$$
A_{0}=0, A_{1}=0, \phi_{1} \equiv W(r), \phi_{2}=0
$$

has a discrete sequence of static solutions. In this sector the ansatz Eq.(2) is equivalent to the usual "monopole" ansatz

$$
W_{0}^{a}=0, \quad W_{i}^{a}=\epsilon_{a i j} \frac{n_{j}}{r}(1-W(r))
$$

with $n_{j}=x_{j} / r$.

The corresponding reduced action is

$$
S^{\mathrm{red}}=-\int d r\left[\frac{r^{2}}{2} \varphi^{\prime 2}+\mathrm{e}^{2 \varphi}\left(W^{\prime 2}+\frac{\left(W^{2}-1\right)^{2}}{2 r^{2}}\right)\right]
$$

The resulting field equations are

$$
\begin{aligned}
W^{\prime \prime} & =\frac{W\left(W^{2}-1\right)}{r^{2}}-2 \varphi^{\prime} W^{\prime} \\
\left(r^{2} \varphi^{\prime}\right)^{\prime} & =2 \mathrm{e}^{2 \varphi}\left(W^{\prime 2}+\frac{\left(1-W^{2}\right)^{2}}{2 r^{2}}\right) .
\end{aligned}
$$


These equations are invariant under a shift $\varphi \rightarrow \varphi+\varphi_{0}$ accompanied by a simultaneous rescaling $r \rightarrow r \mathrm{e}^{\varphi_{0}}$, hence globally regular solutions can be normalized to $\varphi(\infty)=0$.

It was found [1], [2] that Eqs.(8) have a discrete sequence of finite energy solutions $\left\{W_{n}, \varphi_{n}\right\}$, where $n=1,2,3, \ldots$ labels number of zeros of the gauge function $W_{n}(r)$.

The mass of this solution varies from $\approx 0.8$ for $n=1$ to 1.0 for $n \rightarrow \infty$ in natural units $1 / \kappa g$.

The asymptotic behavior of these solutions for $r \rightarrow 0$ is

$$
\begin{aligned}
W_{n}(r) & =1-b_{n} r^{2}+O\left(r^{4}\right), \\
\varphi_{n}(r) & =\varphi_{n}(0)+2 \mathrm{e}^{2 \varphi_{n}(0)} b_{n}^{2} r^{2}+O\left(r^{4}\right),
\end{aligned}
$$

and for $r \rightarrow \infty$ is

$$
\begin{aligned}
W_{n}(r) & =(-1)^{n}\left(1-\frac{c_{n}}{r}+O\left(\frac{1}{r^{2}}\right)\right), \\
\varphi_{n}(r) & =-\frac{d_{n}}{r}+O\left(\frac{1}{r^{4}}\right) .
\end{aligned}
$$

The parameters $b_{n}, \varphi_{n}(0), c_{n}$ and $d_{n}$ have to be determined from the numerical calculations.

Note that at infinity $r \rightarrow \infty$ these solutions are pure gauges (compare Eq.(6))

$$
\frac{\tau^{a}}{2} W_{j}^{a}(\vec{x}) \equiv W_{j}(\vec{x})=-\frac{i}{g}\left(\partial_{j} U\right) U^{-1}
$$

with

$$
U=\mathrm{e}^{-\frac{i}{2} \pi \vec{\tau} \cdot \vec{n}}
$$

for $n$ odd and $U=1$ for even $n$.

\section{Fractional charge}

One can assign a fermion (baryon) number to the dilatonic sphaleron exactly in the same way as for the electroweak sphaleron [6]. It comes out to be $\frac{1}{2}$.

The argument goes as follows. Because of the ABJ anomaly [10] the fermion current is not conserved at the quantum level

$$
\partial_{\mu} j_{B}^{\mu}=\frac{1}{32 \pi^{2}} F_{\mu \nu}^{a} \tilde{F}^{a \mu \nu}
$$

where $\tilde{F}_{\mu \nu}^{a}=\frac{1}{2} \epsilon_{\mu \nu \rho \sigma} F^{a \rho \sigma}$. Change in baryon charge $Q_{B}=\int d^{3} x j_{B}^{0}$ is related to the topological properties of the gauge field configuration. Integrating Eq.(13) and neglecting a possible (integer) contribution from a baryon current at infinity one obtains

$$
Q_{B}\left(t_{2}\right)-Q_{B}\left(t_{1}\right)=\frac{g^{2}}{32 \pi^{2}} \int_{t_{1}}^{t_{2}} d t \int d^{3} x F_{\mu \nu}^{a} \tilde{F}^{a \mu \nu}
$$


It is well known that $F \tilde{F}$ is a total divergence $F_{\mu \nu}^{a} \tilde{F}^{a \mu \nu}=\partial_{\mu} K^{\mu}$ where

$$
K^{\mu}=\epsilon^{\mu \nu \rho \sigma}\left(F_{\nu \rho}^{a} W_{\sigma}^{a}-\frac{1}{3} g \epsilon_{a b c} W_{\nu}^{a} W_{\rho}^{b} W_{\sigma}^{c}\right)
$$

Using this fact one can write

$$
Q_{B}\left(t_{2}\right)-Q_{B}\left(t_{1}\right)=N_{C S}\left(t_{2}\right)-N_{C S}\left(t_{1}\right)+\frac{g^{2}}{32 \pi^{2}} \int_{t_{1}}^{t_{2}} d t \int_{S_{\infty}} d \vec{s} \cdot \vec{K}
$$

where $S_{\infty}$ is the sphere at spatial infinity and

$$
N_{C S}(t)=\frac{g^{2}}{32 \pi^{2}} \int_{x^{0}=t} d^{3} x K^{0}
$$

is the Chern-Simons number of the field at the time $t$.

Now we can consider a time dependent gauge field starting at $t=t_{1}$ at the trivial vacuum (with $N_{C S}=0$ ) and arriving at $t=t_{2}$ at the sphaleron configuration. Assuming that $Q_{B}\left(t_{1}\right)=0$ we obtain

$$
Q_{B}(\text { sphaleron })=N_{C S}\left(t_{2}\right)+\frac{g^{2}}{32 \pi^{2}} \int_{t_{1}}^{t_{2}} d t \int_{S_{\infty}} d \vec{s} \cdot \vec{K}
$$

In the radial gauge $n_{i} W_{i}=0$ the pure gauge configuration Eqs.(11), (12), which the sphaleron (with odd $n$ ) approaches for $r \rightarrow \infty$ is direction dependent. In this gauge $Q_{B}$ picks up its value from the second term in Eq.(18). Since $Q_{B}$ is gauge invariant (up to large gauge transformations) one can choose a more convenient gauge in which the gauge potential $W_{j}$ vanishes faster than $\frac{1}{r}$ and $Q_{B}$ has no contribution from the second term in Eq.(18). For this purpose one has to apply a gauge transformation $\Omega$, which is regular at the origin $r=0$ and removes the direction dependence of $W_{j}$ at infinity. A possible choice for $\Omega$ is

$$
\Omega=\mathrm{e}^{\frac{1}{2} i \Theta(r) \vec{\tau} \cdot \vec{n}}
$$

where $\Theta \in[0, \pi]$. Under this gauge transformation the gauge configuration Eq.(6) goes to

$$
W_{j}^{a} \rightarrow \tilde{W}_{j}^{a}=\frac{W \cos \Theta-1}{g r} \epsilon_{j a k} n_{k}+\frac{W \sin \Theta}{g r}\left(\delta_{a j}-n_{a} n_{j}\right)+\frac{1}{g} \frac{d \Theta}{d r} n_{a} n_{j} .
$$

If $\Theta \rightarrow \pi$ fast enough for $r \rightarrow \infty$ the potential Eq.(20) vanishes faster then $\frac{1}{r}$ and no contribution to $Q_{B}$ from the surface term Eq.(18) arises. Substitution of Eq.(20) into Eq.(18) gives

$$
Q_{B}=\frac{1}{2 \pi}[\Theta(r)-W \sin \Theta(r)]_{0}^{\infty}=\frac{1}{2}
$$


Thus the solution with odd $n$ have $Q=\frac{1}{2}$ and the solutions with even $n Q=0$.

\section{Fermion Zero Mode}

In this section we will analyse the existence of fermion zero modes in the background of the dilatonic sphalerons. The situation is very similar to the one considered in the paper by Gibbons and Steif [15], who analysed the same problem for the BMK solutions.

The Dirac equation for the isodoublet fermions in the external gauge field can be written in the form

$$
i \frac{\partial \Psi}{\partial t}=H_{D} \Psi
$$

with

$$
H_{D}=-i(\vec{\alpha} \cdot \vec{\nabla}-i g \vec{\alpha} \cdot \vec{W}),
$$

and the matrices $\vec{\alpha}$ in a chiral representation are

$$
\vec{\alpha}=\gamma^{0} \vec{\gamma}=\left(\begin{array}{cc}
\vec{\sigma} & 0 \\
0 & -\vec{\sigma}
\end{array}\right)
$$

where $\vec{\sigma}$ are the usual Pauli spin matrices. We want to show that time-independent Dirac equation

$$
H_{D} \Psi=E \Psi
$$

has a normalizable zero energy solution.

The scalar product is given by

$$
\left\langle\Psi_{1} \mid \Psi_{2}\right\rangle=\int d^{3} x \Psi_{1}^{\dagger} \Psi_{2}
$$

One can decompose $\Psi$ into its left and right chiral components

$\Psi=\left(\begin{array}{c}\psi_{L} \\ \psi_{R}\end{array}\right)$. The Dirac equation for the left (right) component becomes

$$
[\vec{\sigma} \cdot \vec{\nabla}-i g \vec{\sigma} \cdot \vec{W}] \psi=0
$$

From the ansatz Eq.(6) it follows that

$$
\vec{\sigma} \cdot \vec{W}=\frac{W-1}{2 g r}(\vec{n} \cdot \vec{\sigma} \times \vec{\tau}) .
$$

Note that the gauge configuration Eq.(6) is invariant under space rotations accompanied by corresponding gauge transformations. This implies that the total angular momentum $\vec{J}=\vec{L}+\vec{S}+\vec{T}$, the sum of orbital angular momentum $\vec{L}$, spin $\vec{S}=\frac{1}{2} \vec{\sigma}$ and isospin $\vec{T}=\frac{1}{2} \vec{\tau}$ is conserved. One can check that $\vec{J}$ commutes with $H_{D}$. Therefore one can label the solutions of the Dirac equation by eigenvalues $j$ and $m$ of $\vec{J}^{2}$ and $J_{z}$. 
In the $s$-wave sector $(j=m=0)$ the most general form of a spherically symmetric wave function contains two radial functions $f(r)$ and $g(r)$ [16]

$$
\psi(r)=f(r) \chi_{1}+g(r) \chi_{2}
$$

where $\chi_{1}$ is the constant spinor satisfying $\chi_{1}^{\dagger} \chi_{1}=1$ and the "hedgehog" property

$$
(\vec{\sigma}+\vec{\tau}) \chi_{1}=0
$$

and $\chi_{2}=\vec{\sigma} \cdot \vec{n} \chi_{1}$. The explicit form of $\chi_{1}$ is $\chi_{1}, \alpha m=\frac{1}{\sqrt{2}} \epsilon_{\alpha m} \alpha$ being Lorentz index and $m$ the isospin index.

Using the "hedgehog" property one can check that

$$
(\vec{n} \cdot \vec{\sigma} \times \vec{\tau}) \chi_{a}=-2 i \epsilon_{a b} \chi_{b}, \quad \text { for } \quad a, b=1,2 .
$$

Having this in mind we obtain a system of first order equations for the radial functions

$$
\begin{aligned}
& \frac{d f}{d r}+\frac{1-W}{r} f=0 \\
& \frac{d g}{d r}+\frac{1+W}{r} g=0 .
\end{aligned}
$$

These equations can be trivially integrated with the result

$$
\begin{aligned}
& f(r)=N \mathrm{e}^{-\int_{0}^{r} \frac{1-W}{r} d r} \\
& g(r)=0
\end{aligned}
$$

where $N$ is a normalization constant. For $r \rightarrow 0$ the function $f_{n}(r) \rightarrow N_{n} \mathrm{e}^{-b_{n} r^{2}}$. For $r \rightarrow \infty$

a) for odd $n f_{n}(r)_{r \rightarrow \infty} \rightarrow \frac{1}{r^{2}}$ and $f_{n}$ is normalizable,

b) for even $n f_{n}(r)_{r \rightarrow \infty} \rightarrow \mathrm{e}^{\frac{c_{n}}{r}}$, hence $f_{n}$ is not normalizable in this case.

\section{Stability analysis}

In this section we will consider the question of linear stability of dilatonic sphalerons. In order to analyse stability one has to consider the spectrum of the small (harmonically) time dependent perturbations in the background solution. Perturbations with negative energy (complex frequency) correspond to exponentially growing modes indicating to the instability of the background solution.

We will consider perturbations of the type

$$
\begin{aligned}
\phi_{1} \rightarrow W(r)+v(r) e^{i \omega t} & \phi_{2} \rightarrow \chi(r) \mathrm{e}^{i \omega t} \\
\varphi \rightarrow \varphi(r)+\psi(r) e^{i \omega t} & A_{1} \rightarrow a(r) e^{i \omega t}
\end{aligned}
$$

Calculation of the quadratic action shows that the $(\chi, a)$ sector decouples from the $(v, \psi)$ sector,

$$
S^{(2)}=S_{(v, \psi)}^{(2)}+S_{(\chi, a)}^{(2)}
$$


where

$$
\begin{aligned}
S_{(v, \psi)}^{(2)} & =\int d r\left(\frac{1}{2} r^{2} \psi^{\prime 2}-\omega^{2}\left(\frac{1}{2} r^{2} \psi^{2}+e^{2 \varphi} v^{2}\right)\right. \\
& \left.+e^{2 \varphi}\left[v^{\prime 2}-4 W^{\prime} v \psi^{\prime}+\frac{3 W^{2}-1}{r^{2}} v^{2}+\left(2 W^{\prime 2}+\frac{\left(W^{2}-1\right)^{2}}{r^{2}}\right) \psi^{2}\right]\right)
\end{aligned}
$$

is the same as in [1] and

$$
S_{(\chi, a)}^{(2)}=\int d r \mathrm{e}^{2 \varphi}\left[\chi^{\prime 2}+\frac{\left(W^{2}-1\right)}{r^{2}} \chi^{2}+W^{2} a^{2}+2 W^{\prime} a \chi-2 W a \chi^{\prime}-\omega^{2}\left(\frac{1}{2} r^{2} a^{2}+\chi^{2}\right)\right]
$$

with $\varphi$ respectively $W$ the background solutions.

The spectrum of the perturbations in the $(v, \psi)$ sector was already analyzed in [1], [2]. For those solutions, which were analysed numerically, $n$ negative modes for the $n^{\text {th }}$ solution were found.

Here we will concentrate on the $(\chi, a)$ sector. The replacement $\chi \rightarrow \mathrm{e}^{-\varphi} \tilde{\chi}, a \rightarrow$ $\mathrm{e}^{-\varphi} \tilde{a}$ brings the action Eq.(37) to the form:

$$
\begin{array}{r}
\tilde{S}_{(\tilde{\chi}, \tilde{a})}^{(2)}=\int d r\left[\tilde{\chi}^{\prime 2}+\left(\varphi^{\prime \prime}+\varphi^{\prime 2}+\frac{\left(W^{2}-1\right)}{r^{2}}\right) \tilde{\chi}^{2}-\omega^{2}\left(\frac{1}{2} r^{2} \tilde{a}^{2}+\tilde{\chi}^{2}\right)\right. \\
\left.+W^{2} \tilde{a}^{2}+2 W^{\prime} \tilde{a} \tilde{\chi}+2 W \varphi^{\prime} \tilde{a} \tilde{\chi}-2 W \tilde{a} \tilde{\chi}^{\prime}\right] .
\end{array}
$$

The equation of motion are:

$$
\begin{aligned}
W\left(\tilde{\chi}^{\prime}-W \tilde{a}\right)-W^{\prime} \tilde{\chi}-W \varphi^{\prime} \tilde{\chi} & =-\frac{\omega^{2} r^{2}}{2} \tilde{a} \\
\left(\tilde{\chi}^{\prime}-W \tilde{a}\right)^{\prime}-\left(\varphi^{\prime \prime}+\varphi^{\prime 2}+\frac{\left(W^{2}-1\right)}{r^{2}}\right) \tilde{\chi}-W^{\prime} \tilde{a}+W \varphi^{\prime} \tilde{a} & =-\omega^{2} \tilde{\chi} .
\end{aligned}
$$

We have performed a numerical analysis of the spectrum for the first few solutions. We find that $n^{\text {th }}$ solution has $n$ negative modes also in $(\chi, a)$ channel. In the Table we have collected our numerical values for the energies $E=\omega^{2}$ of the negative modes of the first three solutions.

\begin{tabular}{|l|l|l|}
\hline$N=1$ & $N=2$ & $N=3$ \\
\hline$E_{1}=-0.2098$ & $E_{1}=-0.1477$ & $E_{1}=-0.1238$ \\
& $E_{2}=-0.0005$ & $E_{2}=-0.0002$ \\
& & $E_{3}=-6 \cdot 10^{-7}$ \\
\hline
\end{tabular}

Tab. Boundstate energies for the $n=1,2,3$ solutions of the YMD theory,

$$
((\chi, a) \text { sector }) \text {. }
$$

Hence altogether we found that the $n^{\text {th }}$ solution of the YMD theory has $2 n$ unstable modes with respect to spherically symmetric perturbations similarly as for the BMK solutions 13 .

\section{Concluding Remarks}

Static solutions of YMD theory are a kind of sphalerons - dilatonic sphalerons. 
In contrast to the electroweak sphaleron in the YMD system one finds solutions with many zeroes of the gauge function.

In conformity with the level crossing picture one finds a normalizable fermion zero mode in the background of these solutions.

These solutions exist in the Einstein-Yang-Mills-dilaton theory as well [17]. Because of the high mass of the solutions the natural situation where they could play a role is in the Early Universe. This question needs further investigation.

\section{Acknowledgements}

I am indebted to P. Breitenlohner, P. Forgács and D. Maison for many stimulating discussions. I would like to thank N. Straumann for the kind hospitality at the University of Zurich, where this work was completed. This work was supported in part by Tomalla Fellowship.

\section{References}

[1] G. Lavrelashvili and D. Maison, Phys. Lett. B295 (1992) 67.

[2] P. Bizon, Phys. Rev. D47 (1993) 1656.

[3] R. Bartnik and J. McKinnon, Phys. Rev. Lett. 61 (1988) 141.

[4] R.F. Dashen, B. Hasslacher, and A. Neveu, Phys. Rev. D10 (1974) 4138.

[5] N. Manton, Phys. Rev. D28 (1983) 2019.

[6] F.R. Klinkhamer and N.S. Manton , Phys. Rev. D30 (1984) 2212.

[7] M.S. Volkov and D.V. Gal'tsov, Phys. Lett. B273 (1991) 255.

[8] R. Jackiw and C. Rebbi, Phys. Rev. Lett. 37 (1976) 172; C.G. Callan, R.F. Dashen, and D.J. Gross, Phys. Lett. 63B (1976) 334.

[9] G. 't Hooft, Phys. Rev. Lett. 37 (1976) 8; Phys. Rev. D14 (1976) 3432.

[10] S.L. Adler, Phys. Rev. 177 (1969) 2426;

J.S. Bell and R. Jackiw, Nuovo Chimento 60A (1969) 47.

[11] V.A. Matveev, V.A. Rubakov, A.N. Tavkhelidze, and M.E. Shaposhnikov, Sov. Phys. Usp. 31 (1988) 916.

[12] P. Breitenlohner, P. Forgács and D. Maison, Commun. Math. Phys. 163 (1994) 141.

[13] G. Lavrelashvili and D. Maison, A remark on the instability of the BartnikMcKinnon solutions, Preprint MPI-PhT/94-64, hep-th/9409185.

[14] E. Witten, Phys. Rev. Lett. 38 (1977) 121;

P. Forgács and N.S. Manton, Commun. Math. Phys. 72 (1980) 15.

[15] G.W. Gibbons and A.R. Steif, Phys. Lett. B314 (1993) 13.

[16] R. Jackiw and C. Rebbi, Phys. Rev. D13 (1976) 3398.

[17] G. Lavrelashvili and D. Maison, Nucl. Phys. B410 (1993) 407. 